\title{
Application of Mandibular Nerve Blocks by Dental Practitioners in Bulgaria with a Working Experience of More than 10 Years - Survey
}

\author{
Rusi Argirov*, Petia Pechalova \\ Department of oral surgery, Faculty of dental medicine, Medical University of Plovdiv, Bulgaria \\ *Corresponding Author: Rusi Argirov, Department of oral surgery, Faculty of dental medicine, Medical \\ University of Plovdiv, Bulgaria, Email: russsi144@gmail.com
}

\begin{abstract}
There are many different techniques for mandibular nerve block anesthesia. The purpose of this article is to research the usage of mandibular nerve blocks from the dental practitioners with work experience more than 10 years in Bulgaria. An anonymous survey was filled by 564 dental physicians.

Most of the dentists stated that they use mandibular nerve block in their daily practice ( $63.12 \%-n=356)$, while $32.45 \%(n=183)$ stated that they employ these type of techniques in some cases. Only $4.43 \%(n=25)$ of the filled questionnaires contained information about dentists that don't use mandibular nerve blocks. The most common technique was the Weisbrem's- $60.46 \%(n=341)$, the second most used was Halsted's $34.04 \%(n=192)$ and only a handful of practitioners use the Gow-Gates block - 0.89\% (n=5) and the Vazirani-Akinosi- $0.18 \%(n=1)$. Most of the dentists that participated of the survey asses their nerve blocks as successive (fast onset, providing good pain control) - $88.65 \%(n=500), 4.26 \%(n=24)$ asses their techniques as such with slow onset and requiring supplemental injection for a painless procedure and $2.66 \%$ $(n=15)$ state that their mandibular nerve blocks don't provide any pain control and should be replaced by another type of local anesthesia.
\end{abstract}

\section{INTRODUCTION}

Mandibular nerve blocks are frequently used in the general dental practice. There are many alternative techniques to the classical Halsted approach to the IANB, which were thoroughly researched and proved its usefulness. The choice of the clinician, as to what technique to use, is heavily dependent on many different factors, for example - the success rate of the technique as well as the complication that may arise from it. All doctors of dental medicine should be well versed in different techniques of mandibular nerve blocks, so that they can effectively choose the most appropriate for the concrete clinical case. Many of the IANB techniques require from the clinician to identify intraoral landmarks, which in most of the cases is really difficult, because of their high variability. Because of the many anatomic variation, and the difficulty in their clear identification, the success rate in some cases can be really low and the learning curve of the different techniques can be quite hard.

The purpose of this article is to acquire data about the usage of mandibular nerve blocks on the territory of the Republic of Bulgaria and more precisely by the clinicians with working experience above 10 years.

\section{METHODS}

An anonymous survey, which contained 15 questions about the applications of mandibular nerve blocks, the usage of different techniques for IANB and the clinical situations in which they were used, was distributed to dental practitioners with an experience of more than 10 years. The questionnaire was made with Google Forms, and links were sent to the participants. The questionnaire was also distributed by paper variants. Till the end of the chooses period, 564 surveys were filled and returned. The results were processed and statistically analyzed with IBM SPSS 23 and Microsoft Excel.

\section{Results}

From all the participants in the study, $16.66 \%$ $(n=94)$ had a dental specialty. The largest part of the specialists was in the field of Prosthetic Dentistry $-38.30 \% \quad(n=36)$, followed by the specialists in Orthodontics - $19.15 \% \quad(\mathrm{n}=18)$, Conservative dentistry and endodontic -23.40 
$\%(\mathrm{n}=22)$, Oral surgery $-12.77 \%(\mathrm{n}=12)$ and Periodontics $-1.06 \% \quad(n=1)$. Two of the clinicians stated that they have two specialties $(2.17 \%)$.

In the question about the workplace of the participants, most of the dental practitioners with experience of more than 10 years, had their own practice- $76.77 \% \quad(n=433)$. They were followed by the clinicians that work in a dental practice with two dentist, who work independently from each other $-12.94 \%(n=73)$, then were the dentist that work in a group practice with more than two practitioners $8.87 \%(\mathrm{n}=50)$, the ones that work in a faculty of dental medicine, medical university - $1.24 \%$ $(n=7)$, and in last place were the ones that practiced in a hospital/maxillo-facial department $-0.18 \%(n=1)$. (Figure 1)

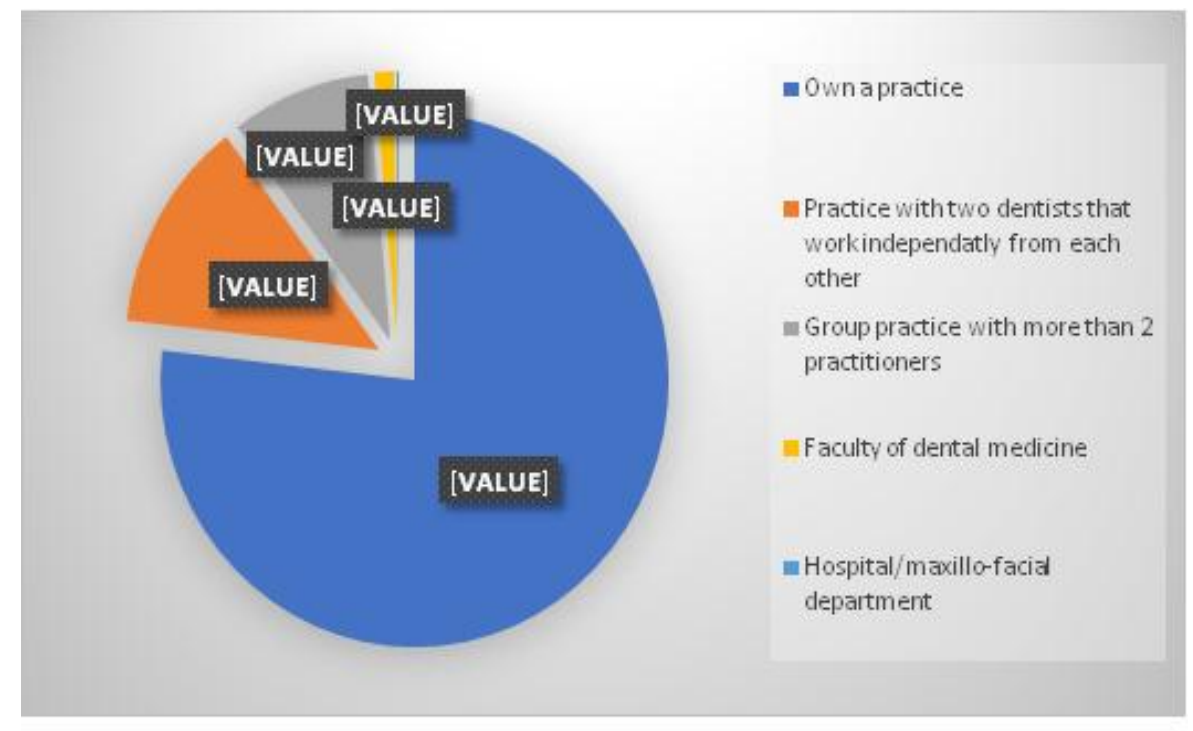

Fig1: Distribution of the dental practitioners with experience of more than 10 years by the type of their workplace.

When we are on the subject of frequency, most of the dental practitioners with an experience of more than 10 years $-63.12 \%(n=356)$ claim that they occasionally use mandibular nerve blocks, following them are the ones that frequently use nerve block anesthesia on the mandible in their daily practice $-32.45 \%(n=183)$, and only $4.43 \%$ $(n=25)$, indicated that they don't employ blocks in their practice. (Figure 2)

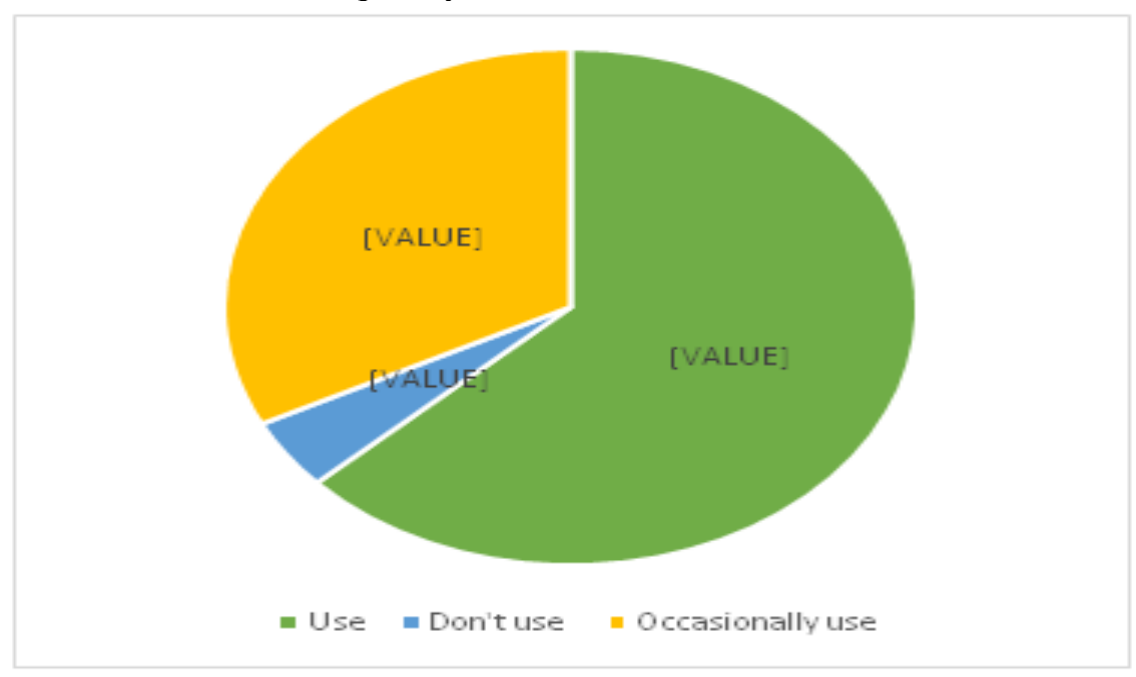

Fig2: Distribution of the participants depending on whether or not they use mandibular nerve blocks.

Most of the participants in the study claimed that they use mandibular nerve blocks, during single tooth interventions as well as procedures that involve multiple teeth $-87.06 \%(\mathrm{n}=491)$. Only $8.51 \% \quad(\mathrm{n}=48)$ indicated that they use mandibular nerve blocks only during interventions involving multiple teeth. No participants answered that they use mandibular nerve blocks for interventions on a single tooth only. (Figure 3) 


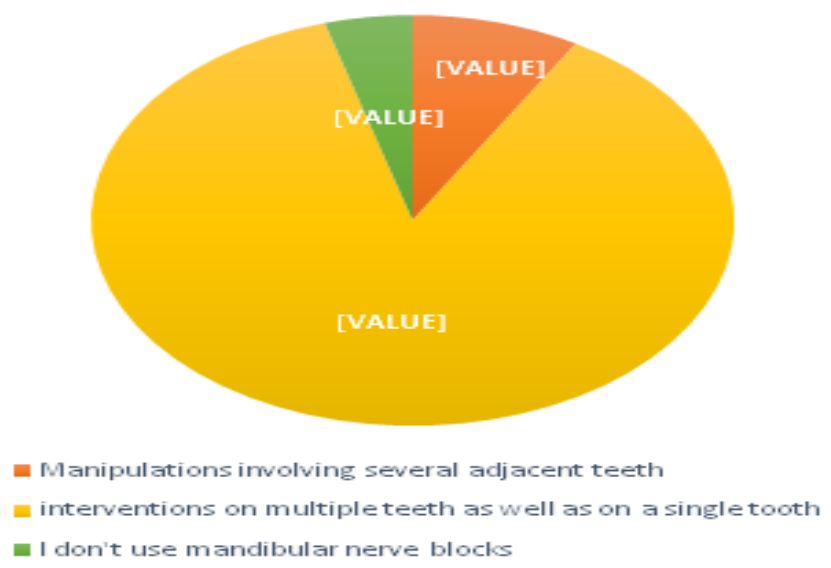

Fig3: Clinical situations in which dental practitioners prefer to use mandibular nerve block anesthesia.

From all the surveyed clinicians $60.46 \%$ $(n=341)$ preferred the Weisberg approach (Torusal nerve block of the mandible), followed by the dental practitioners that more frequently use the Halsted approach to IANB - 34.04\% $(n=192)$. Gow-Gates was the preferred method for only $0.89 \%(n=5)$ of the dentists and the Akinosi technique was used only by $-0.18 \%$ $(\mathrm{n}=1)$-figure 4.

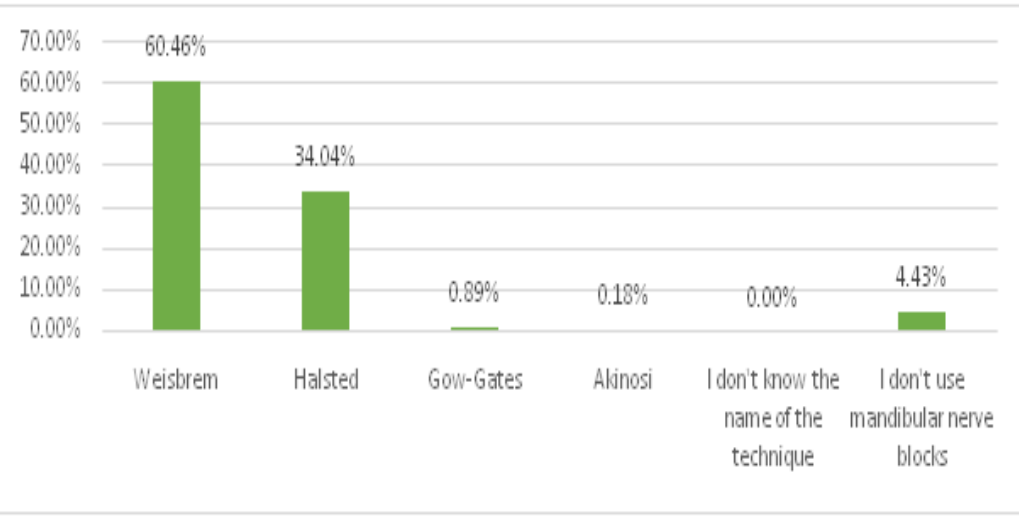

Fig4: Mandibular nerve block methods of choice for the dentists that participated in the study.

The largest part of all the dentists that participated - $88.65 \%(n=500)$ assessed their blocks as successful enough (fast onset and profound enough anesthetic effect). Only $4.26 \%$ $(n=24)$ thought that their nerve blocks didn't provide enough anesthesia and needed to use a supplementary technique. Even fewer dentists $2.66 \%(\mathrm{n}=15)$ claimed that their nerve blocks on the mandible were ineffective and needed to substitute the technique with another method. (Figure 5)

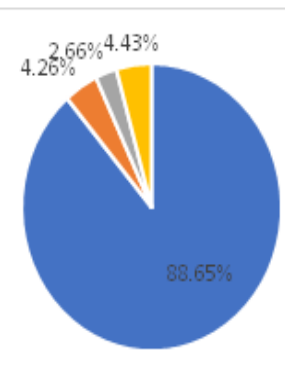

- Profound enough anesthesia with a fast onset

- lacking pain control and need for a suplementary technique

॥ Failure to anesthetize the tissues so substitution with another method is requeired

"I don't use mandibular nerve blocks

Fig5: Assessment of the effect of the mandibular nerve blocks of the participants. 


\section{DISCUSSION}

Most of the clinicians in our study rely on the Weisberg approach to anesthetize the inferior alveolar nerve. These dentists are followed by the group that would rather use the classical Halsted method. Only $0.89 \%(n=5)$ and $0.18 \%$ $(n=1)$ claimed that they prefer the Gow-Gates and the Akinosi-Vazirani techniques accordingly. In a study, done by Maryam AlHindi [1], 10.3\% from all the surveyed claimed that they are well versed in the GowGates mandibular nerve block and only $2.9 \%$ claim that they are familiar with the AkinosiVazirani closed-mouth nerve block. In the same study 22 of all the 238 surveyed dentists indicated that they have eno/ugh theoretical knowledge and practical skills to use alternative to the classical Halsted approach to inferior alveolar nerve block. A reason for the young clinicians to avoid using the Gow-Gates technique can be the greater need for extensive anatomical and technical skills of the operator. The study of Manish Dubey [2] and coll. compares the Halsted approach and the GowGates technique. In the study, there are two groups of 50 patients corresponding to the two mandibular nerve blocks, which were used. In the Gow-Gates group, there were 11 failures to anesthetize the patients, when 1 cartridge was used and there was a need for another injection to be made. On the other hand in the Halsted group, there were only two cases of failure.

The authors of the study reckon that the high number of failures in the Gow-Gates group were because of the inaccurate deposition of the anesthetic. Another problem of the Gow-Gates technique is the slow onset of the anesthesia. In the study by Manish Dubey [2], the onset of anesthesia in the Gow-Gates was on average between 11 and 13 minutes, while in the Halsted group the onset of anesthesia occurred on average between 4 and 5 minutes after the application of the anesthetic agent. According to the author this is happening because of the larger diameter of the nerve trunk in the region of the neck of the condyle, and because of that the anesthetic needs more time to diffuse through. According to literature the Gow-Gates and the Akinosi-Vazirani nerve block have some advantages to the classic approach to the IANB (Halsted technique). Diandian Li [3] researched the three different mandibular nerve blocks and compared the positive aspiration rate. In the 420 patients that participated, there were no positive aspirations, when the Gow-
Gates and the Akinosi-Vaziraniwere used, but there were 23 positives, when the Hasted technique was applied. In his study, Jiacai He [4] established that only one of his patients from the Akinosi group had a positive aspiration, while here were 4 patients from the Halsted group that also gave a positive probe. Moreover, Jizhong Lv [5] also reported no positive aspirations in patients that received the Akinosi block and there were 6 people that suffered from a positive aspiration, while receiving the Halsted approach. Martinaz-G [6] also reports that in his study there was only one patient with positive aspiration from the Akinosi technique, while there were 6 patients with the Halsted technique that experienced positive aspiration. Fei-Wang [7] also reaches the same conclusion as in his study there were only 1 patient with positive aspiration from the Gow-Gates group and 3 from the Halsted group. The results from these studies were processed in a meta-analysis by Fanyuan $\mathrm{Yu}$ [9] and coll. and they show that there is a statistically lower chance to get a positive aspiration, when using the Gow-Gates or Akinosi technique, than during the application of the classic Halsted method.

\section{CONCLUSION}

The dental practitioners in Bulgaria with a clinical experience of more than 10 years prefer the Torusal mandibular nerve block (Weisberg technique) and the classical Halsted approach. It is of great importance for the dentists in Bulgaria to improve their knowledge and practical skills in the alternative mandibular nerve blocks.

\section{REFERENCES}

[1] Maryam AlHindi, Bayan Rashed, NouraAlOtaibi. Failure rate of inferior alveolar nerve block among dental students and interns. Saudi Med J 2016; Vol. 37 (1): 84-89.

[2] Manish Dubey, Iqbal Ali, Deepak Passi, Anil kumaryadav, Devesh Tiwari, Gaurav kumarSaha, Lokesh Chandra and Anchal Srivastava. Comparative Evaluation of Classical Inferior Dental Nerve Block and Gow-Gates Mandibular Nerve Block for Posterior Dentoalveolar Surgery: A prospective study and literature review. Ann Med Health Sci Res. 2017; 7: 92-96

[3] Li, D., Nan, X. \& Xie, G. Clinical application and evaluation of three types of blockanesthesia of inferior alveolar nerve. Chinese Journal of Practical Stomatology 2, 36-38 (2009)

[4] He, J. \& Jiang, C. Evaluation of two different methods of inferior alveolar nerve block 
anesthesia. Acta Universitatis Medicinalis Anhui 35, 281-282 (2000).

[5] Lv, J. \& Zhang, C. Clinical application and evaluation of two types of block anesthesia. Journal OfHeze MedicalCollege 21, 3-5(2009).

[6] Martínez-González, J. M., Benito-Peña, B., Fernández-Cáliz, F., SanHipólito-Marín, L. \& Peñarrocha-Diago, M. A comparative study of direct mandibular nerve block and the Akinosi technique. Med Oral 8, 143-149 (2003).

[7] Wang, F. \& Feng, C. Applying Gow-Gates mandibular nerve conduction anesthesia to extraction of mandible impacted tooth.J Modern Stomatol 16, 69-70 (2002).

[8] Yang, J., Liu, W. \& Gao, Q. The anesthetic effects of Gow-Gates technique of inferior alveolar nerve block in impacted mandibularthird molar extraction. West China Journal of Stomatology 31, 381-384 (2013).

[9] Fanyuan, Y. et al. Evaluation of Three Block Anesthesia Methods for Pain Management During Mandibular Third Molar Extraction: A Meta-analysis. Sci. Rep. 7, 40987; doi: 10.10 38/srep40987 (2017).

Citation: Rusi Argirov, Petia Pechalova, Application of Mandibular Nerve Blocks by Dental Practitioners in Bulgaria with a Working Experience of More than 10 Years - Survey.ARC Journal of Surgery.2019; 5(4):8-12. DOI: http://dx.doi.org/10.20431/2455-572X.0504002

Copyright:@ 2019 Authors. This is an open-access article distributed under the terms of the Creative Commons Attribution License, which permits unrestricted use, distribution, and reproduction in any medium, provided the original author and source are credited. 